\title{
Extraosseous Ewing's sarcoma arising from the pterygomandibular space
}

\author{
Wei Li, Jian Tao Huang, Xiao Qing Chen, Rong Hua Shi, Lei Jiang, Yun Fu Zhao*
}

Department of Stomatology, Changzheng Hospital, Second Military Medical University, Shanghai, China. Email: *zhaoyf1818@126.com

Received 17 July 2011; revised 25 August 2011; accepted 3 September 2011.

\begin{abstract}
Extraosseous Ewing's sarcoma (EES) arising in head and neck is extremely rare. We report on a rare case of EES originating from the pterygomandibular space. A 15-year-old boy was seen with a rapidly growing mass in the right upper neck, difficulty in opening mouth, dysphagia, numbness in the right lower lip and buccal skin. Clinical and radiological examinations indicated that a soft tissue mass originated from the pterygomandibular space with the submandibular space and mouth floor extension. Histological and immunohistochemical evaluations of the biopsy specimen revealed poorly differentiated small blue round cells with positive stains for CD99 and neuron-specific enolase. According to the clinical manifestation, CT and MRI findings, histological pattern and the results of the immunohistochemical studies, the final diagnosis was EES. Our patient was treated with chemotherapy and radiotherapy. The lesion recurred locally after 10 months and he died of multiple distant metastases 22 months later. Early and confident diagnosis coupled with combined surgical excision and modern chemotherapy/radiotherapy appears to be the most effective treatment plan.
\end{abstract}

Keywords: Extraosseous Ewing's Sarcoma; Pterygomandibular Space; Diagnosis; Radiotherapy

\section{INTRODUCTION}

Ewing's sarcoma (ES) of bone was first described by James Ewing in 1921 [1]. ES most commonly arises in the skeleton of adolescents and young adults, and about $15 \%$ are extraosseous. This rare subset of ES, known as extraosseous Ewing's sarcoma (EES), was first reported by Tefft et al. in 1969 [2]. It arises in soft tissue and shares histological, immunohistochemical, and molecular findings with bone ES. EES occurs predominantly in adolescents and young adults between the ages of 10 and 30 years, and follows an aggressive course, with a high recurrence and metastasis rate. In a clinicopathological study of 39 cases of EES reported by Angervall and Enzinger [3], the paravertebral region was the most frequent site of involvement, followed by the lower extremity and chest wall.

EES arising in the head and neck is extremely rare. Although it has been reported in the orbit [4], scalp [5], face [6], nasal cavity [7], paranasal sinus [8], nasopharynx [9], parapharyngeal space [10,11], larynx [12], hard palate [13], submandibular gland [14], parotid gland [15], thyroid gland [16] and soft tissue of the neck [17], to our knowledge, there has been no documented case regarding involvement of the pterygomandibular space by EES. This article reports a rare case of EES originating from the pterygomandibular space with the submandibular space, mouth floor extension and describes the clinical, radiological, histopathological and immunohistochemical features.

\section{CASE REPORT}

In June 2009, a 15-year-old boy presented to our department with a 3-month history of a mass in the right upper neck that had rapidly increased in size. One month before seeking treatment, he had developed difficulty in opening mouth, dysphagia, numbness in the right lower lip and buccal skin. His personal history and his family history were not contributory, and no apparent history of trauma around the right upper neck was noted.

Clinical examination was remarkable for the right submandibular mass measuring $5 \times 7 \mathrm{~cm}$ (Figure 1(a)) The mass was rubbery, mild tenderness, and fixation to underlying structures. The overlying skin was of normal texture and color. No palpable cervical lymphadenopathy was present. Maximal mouth opening was $20 \mathrm{~mm}$. The intraoral examination revealed a large submucosal bulge in the floor of mouth, pushing the right tongue oppositely, however, the tongue motion was preserved. The swelling was covered with ulcerated-appearing 
mucosa (Figure 1(b)). All lower right teeth tested vital to electrical pulp testing. Chest radiograph and abdominal ultrasonography were normal. The laboratory data, including the alkaline phosphatase and lactic acid dehydrogenase level, were within normal limits.

Computed tomography (CT) examination showed a well-circumscribed solid mass, measuring about $9 \times 7 \times$ $9 \mathrm{~cm}$, occupying the right pterygomandibualr, submandibular space and mouth floor, which exhibited heterogeneous post-contrast enhancement. Localized erosion and periosteal reaction of the lingual cortex of the mandible was also noted on CT scan. No evidence was found of calcification, lytic appearance or medullary involvement (Figure 1(c)). Magnetic resonance imaging (MRI) examination was performed to demonstrate the extension of the lesion and surrounding tissue involvement. $T_{1}, T_{2}$ and contrast-enhanced $\mathrm{T}_{1}$-weighted images indicated that a well-circumscribed soft tissue mass closely attached to the lingual cortex of the ascending ramus and extension into the pterygomandibular, submandibular space and mouth floor (Figure 1(d)). The mass was hypointense to isointense on $\mathrm{T}_{1}$-weighted and heterogene-

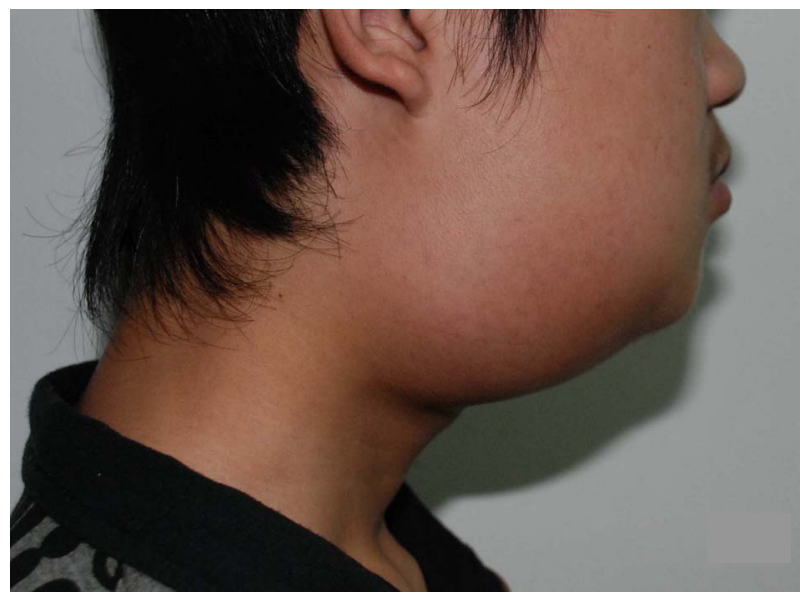

(a)

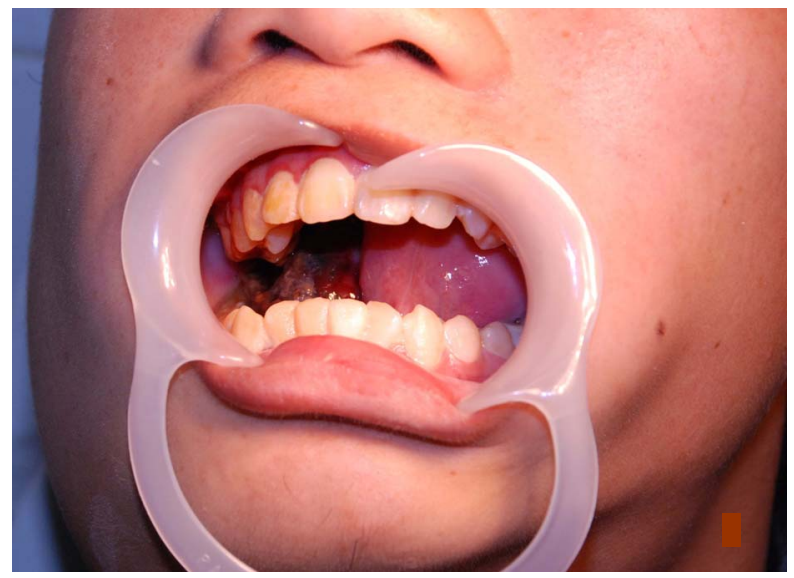

(b)

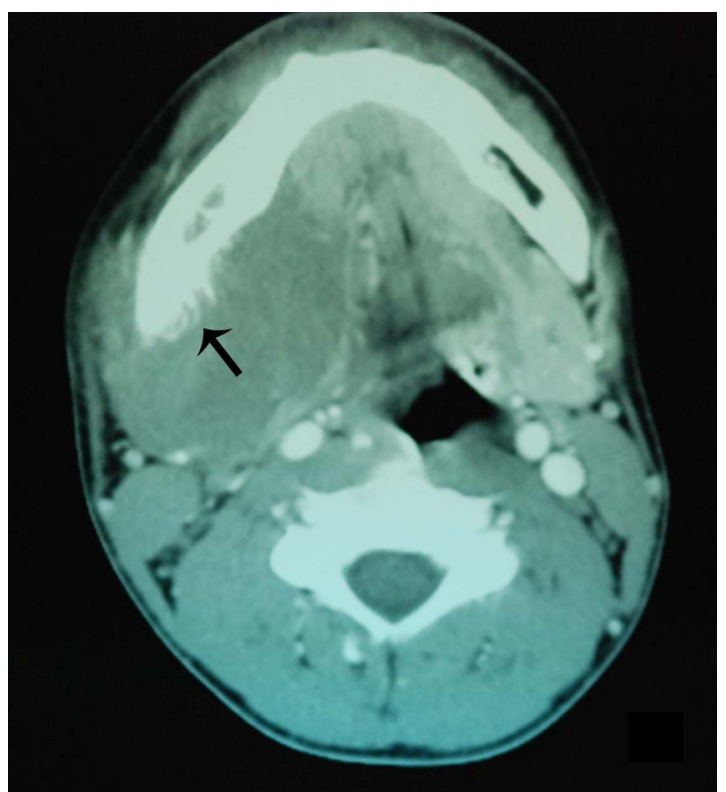

(c)

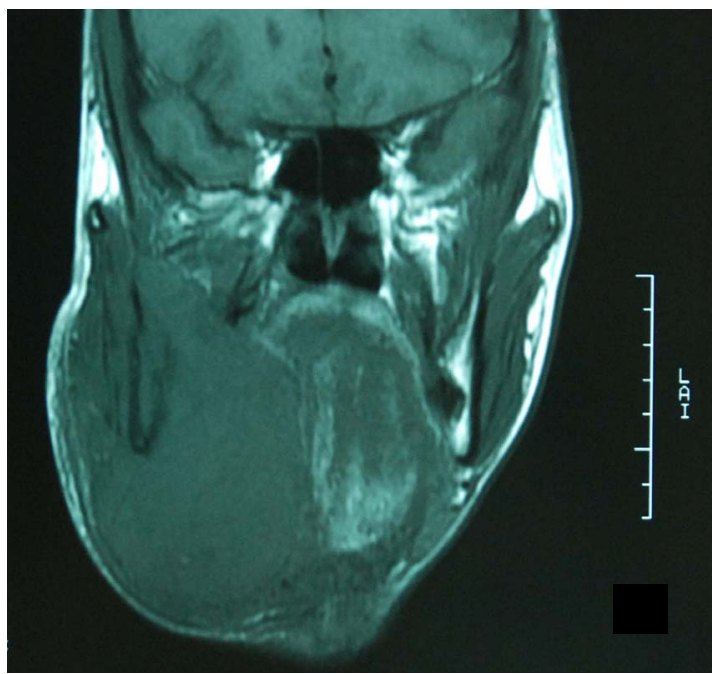

(d)

Figure 1. (a) Clinical photography of the patient at time of initial presentation. Notice the large swelling in the right submandibular region. (b) Intraoral examination showing a large submucosal bulge with ulcerated-appearing mucosa in the right mouth floor. (c) Axial contrast-enhanced (soft tissue window) computed tomography scan showing a well-defined, with heterogeneous contrast-enhanced mass, partially encircling cortex of bone. A variable periosteal "sun-ray" reaction (arrow) of the lingual cortex of the mandible can be seen. No signs of lytic or medullary involvement are seen. (d) Coronal $\mathrm{T}_{1}$ weighted MRI showing a well-circumscribed soft mass, occupying the right pterygomandibualr, submandibular space and mouth floor.

ously hyperintense on $\mathrm{T}_{2}$-weighted scans. On contrast-enhanced $\mathrm{T}_{1}$-weighted images, the lesion showed heterogeneous signal increase with internal hypointense necrotic areas.

In view of the imaging findings, a submandibular in- 
cisional biopsy was carried out. At surgery, the lesion was found to be tightly enveloped in a thin layer of pseudocapsule. Histopathologic examination of the biopsy specimen revealed poorly differentiated small blue round cells with oval nuclei and scanty cytoplasm (Figure 2(a)). A panel of immunohistochemical stains was performed. The tumor cells were positive for CD99 (Figure 2(b)) and neuron-specific enolase (NSE) (Figure 2(c)) and negative for leukocyte common antigen (LCA), CD30, CD20, myosin, actin, and myoglobulin, neurofilament (NF), and the S-100 protein, vimentin, synaptophysin (Syn). The CD99 stain exhibited strong membranous staining. The clinical manifestation, CT and MRI findings, histological pattern and the results of the immunohistochemical studies were compatible with EES.

According to the extent of the tumor and particular anatomy site, the patient's parents refused radical surgery. Chemotherapy and radiotherapy were planned. At first the patient was treated with one cycle of multiagent chemotherapy (cyclophosphamide $750 \mathrm{mg}$, adriamycin $50 \mathrm{mg}$ and vincristine $2 \mathrm{mg}$, actinomycin D $0.6 \mathrm{mg}$ ) and followed by radical radiotherapy. The total dose of 7000 cGy was delivered in 200 cGy fractions, five times per week. The patient appeared to tolerate chemotherapy well, achieving some resolution of swelling in the upper neck and oral cavity. With the subsequent radiotherapy the patient seemed to acquire complete remission. The patient remained well for 10 months with no evidence of recurrence in the upper neck and oral cavity. Unfortunately, MRI examination revealed that the lesion had recurred locally and was located at the lingual bone cortex surface of the ascending ramus (Figure 3) and bone scan showed multiple bone metastases (right femur and T11, 12 vertebrae). Therefore, a second round of focal radiotherapy and combination chemotherapy regimen were discussed with his parents, but they did not accept. He died of widespread metastases to the bones and lungs 22 months after radiotherapy.

\section{DISCUSSION}

There has been remarkable evolution in the concepts regarding ES histogenesis and relation with other small round cell tumors, including peripheral primitive neuroectodermal tumor (pPNET). The relationship between EES and pPNET represents one of the most fascinating controversies in pathology. Both EES and pPNET show varying degree of neuroectodermal differentiation [11]. The term EES has been used for those tumors that lack

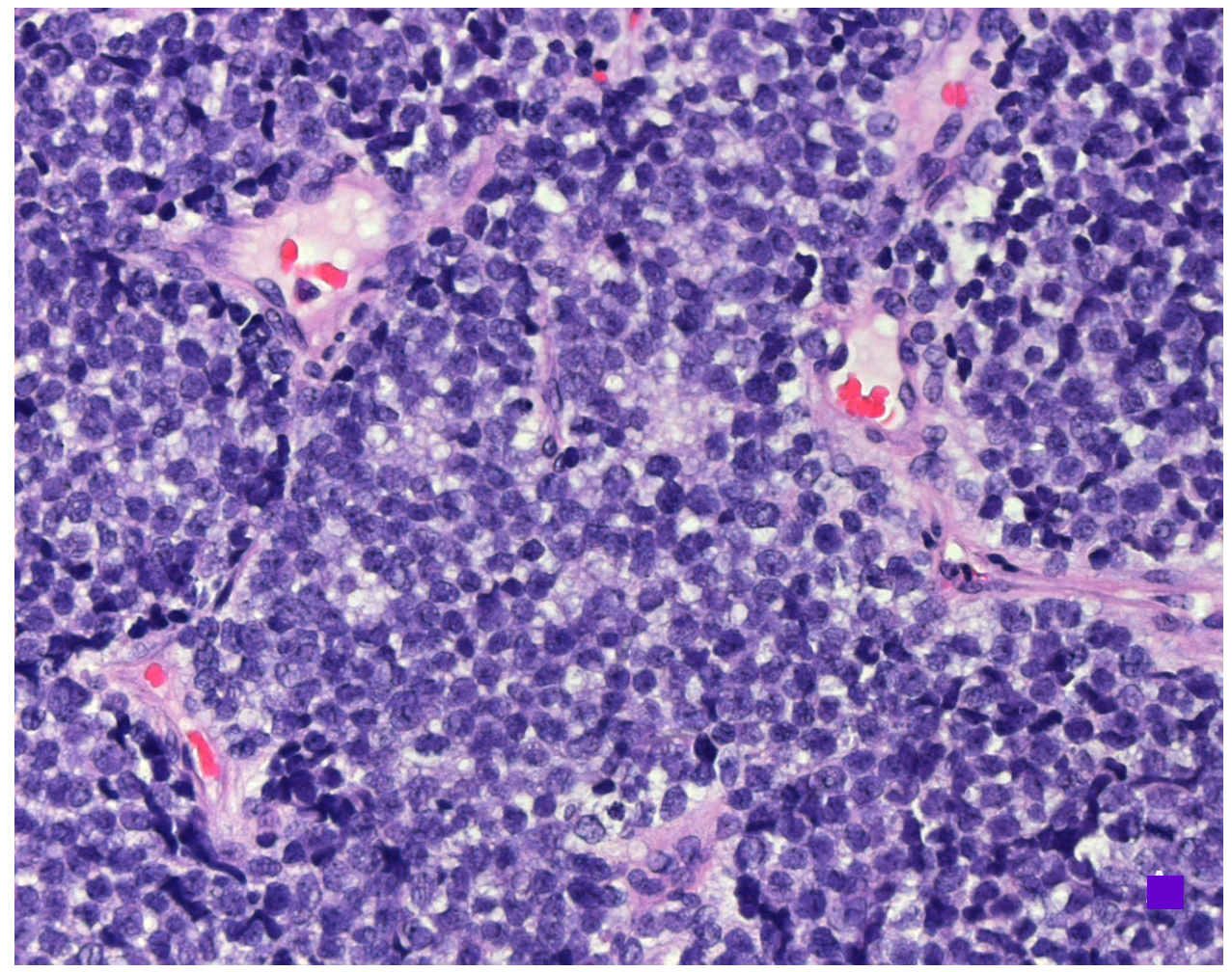

(a) 


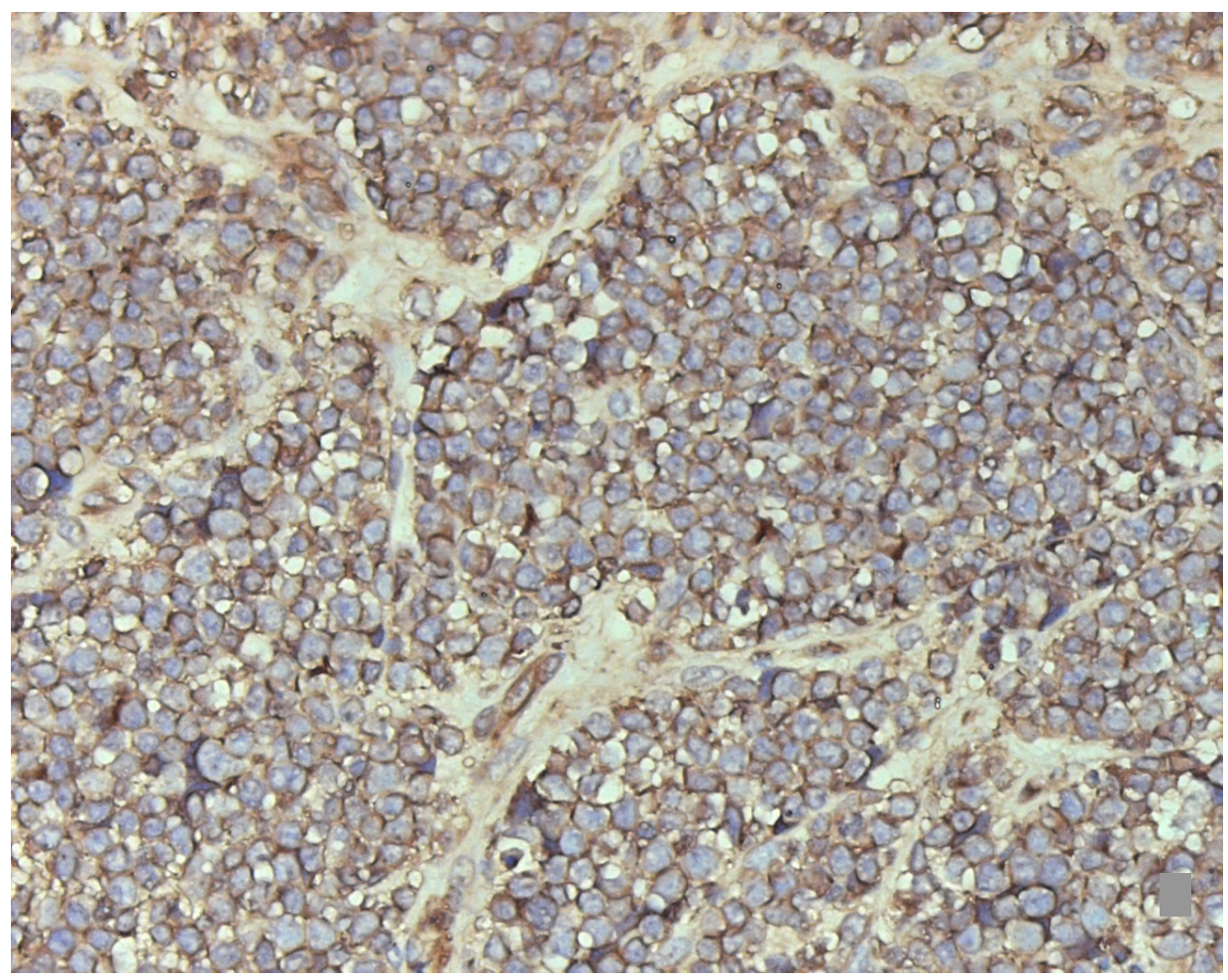

(b)

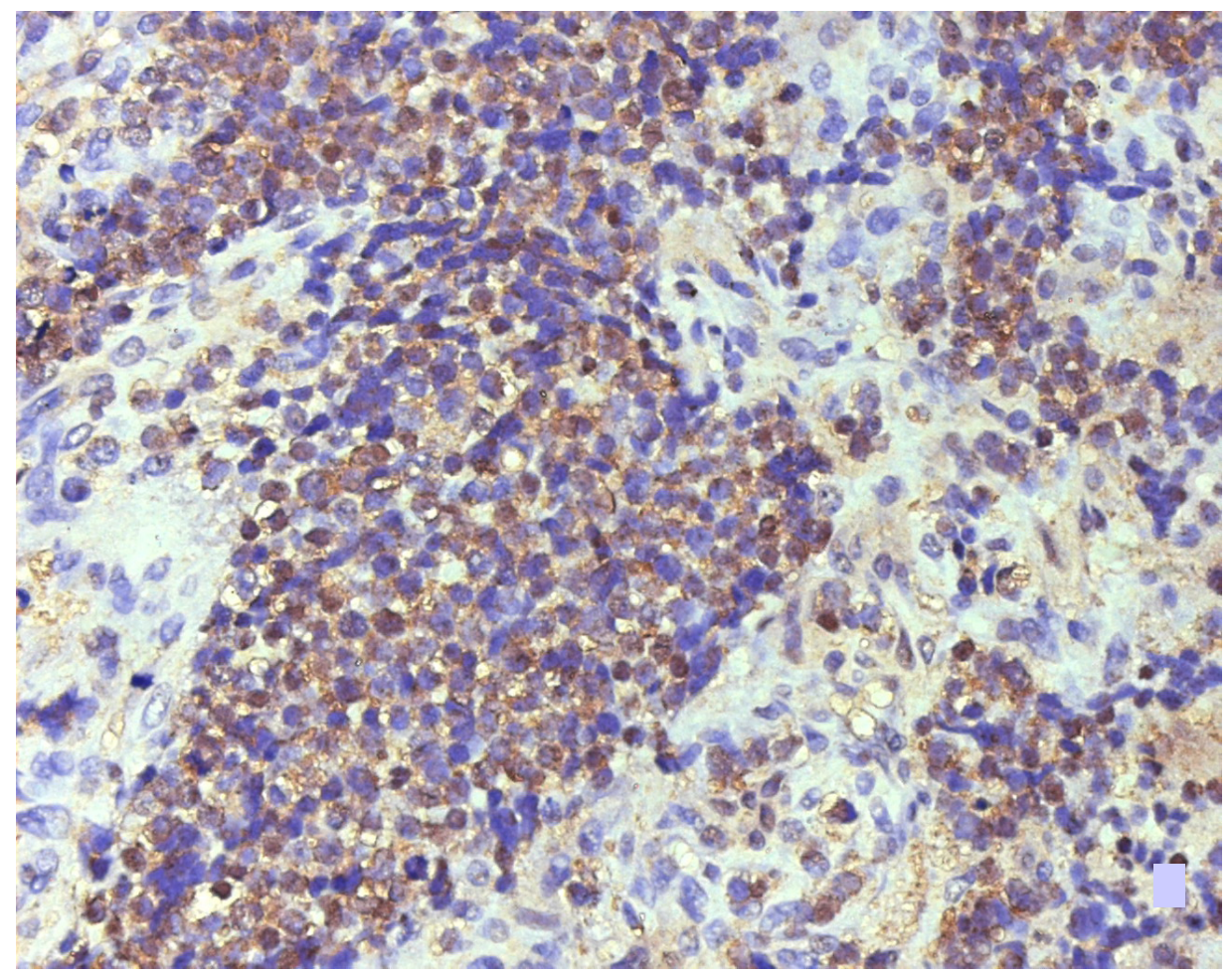

(c)

Figure 2. (a) Photomicrograph showing compactly arranged small blue and round undifferentiated cells with great uniformity (hematoxylin-eosin stain, original magnification $\times 40$ ). (b) The tumor cells showing intense membranous staining with CD99 antibody (original magnification $\times 40$ ). (c) Many tumor cells showing positive staining for antibodies against neuron-specific enolase (original magnification $\times 40$ ). 


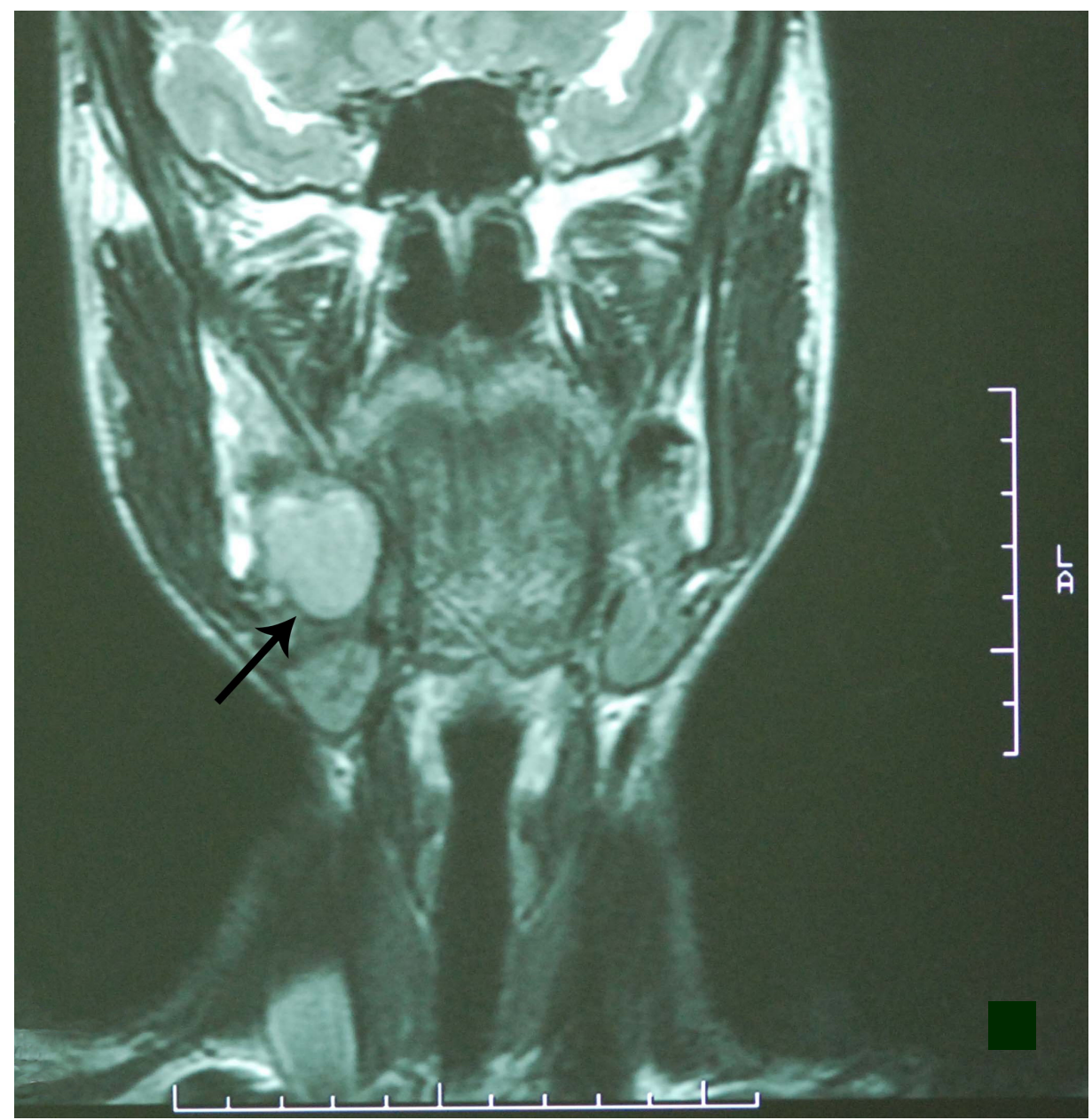

Figure 3. Coronal $\mathrm{T}_{2}$ weighted MRI showing a recurrent lesion (arrow) on outer surface of lingual cortex of the right ascending ramus.

neuroectodermal differentiation by light microscopy, immunohistochemistry and electron microscopy [18]. pPNET shows neuroectodermal differentiation, with rosette formation and immunohistochemical staining for nucleus including neuron specific enolase (NSE), neurofilament (NF) and synaptophysin (Syn) .Currently they are categorized into a group known as the Ewing sarcoma family of tumors (ESFT) which includes classic ES of bone, EES, Askin tumors of the chest wall and pPNET of bone or soft tissues. ESFT exhibits a unique karyotypic abnormality of $\mathrm{t}(11 ; 22)$ (q24; q12) translocation, resulting in the expression of a new chimeric EWS-FLI1 protein. This genetic abnormality can be identified in nearly $90 \%$ of ESFTs. Expression of the MIC2 gene in a majority of these tumors is important from a diagnostic point of view. Although MIC2 is not specific for ESFTs and is expressed in a wide range of unrelated tumors, stain with CD99 (product of the MIC2 gene) provides support for the diagnosis of these tumors, if used with other features. Identification of the fusion transcripts EWS/FLI1 or EWS/ERG by RT-PCR or fluorescent in situ hybridization serves as a sensitive and specific diagnostic test for ESFT [19].

It is not easy to diagnose EES by focusing only on histologic studies, due to its similarity to the rest of the small round cell tumors. Immunohistochemical stains, especially CD99, improve the diagnostic certainty. CD99 is a cell surface glycoprotein found in virtually all ESFTs. Although some other small round cell tumors such as neuoblastoma, lymphomas, and embryonic and alveolar rhabdomyosarcoma may also occasionally show positive staining for CD99, they are excluded by other immunohistochemical stains. Lymphoblastic lymphoma and rhabdomyosarcoma can be excluded by negative staining for LCA, CD30, myosin, actin, and myoglobulin. Neuroblastoma can be excluded by negative staining for NF, NSE, and the S-100 protein. Lack of neurosecretory type granules (NFs) separates classic ES from pPNET [20]. In addition, the majority of rhabdomyosarcomas, neuroblastomas, and osteosarcomas are ane- 
uploid, whereas Ewing's sarcomas are usually diploid [21]. In our case, tumor cells showed no rosette formation and were negative on immunohistochemical stains for LCA, CD30, CD20, myosin, actin, and myoglobulin, NF, S-100 protein, vimentin, Syn. The tumor cells only showed positive staining for CD99 and NSE. Our patient was diagnosed with EES based on the results of extensive immunohistochemical staining as described above.

EES arising in head and neck is extremely rare and only some cases have been reported in the orbit, scalp, face, nasal cavity, paranasal sinus, nasopharynx, parapharyngeal space, larynx, hard palate, submandibular gland, parotid gland, thyroid gland, and soft tissue of the neck [4-17]. However, no case originating from the pterygomandibular space has been reported to date. Clinically, about $75 \%$ of the patients with EES present with a rapidly enlarging swelling which is usually less painful than its osseous counterpart, 30\% patients exhibiting distant metastasis at the time of diagnosis [22]. It has a high propensity to spread locally, infiltrating surrounding fascial planes and invading muscle and bone. Our patient presented with a rapidly growing mass in the submandibular region and oral cavity associated with mild tenderness, restriction of mouth opening, dysphagia, numbness in the right lower lip and buccal skin but did not have any signs of distant metastasis. These clinical findings suggested that the internal pterygoid muscle and mandibular branch of the fifth cranial nerve had been involved in the tumor spread.

CT and MRI are frequently used in the radiological evaluation of ESFTs of the head and neck. However, these modalities cannot provide a specific diagnosis, but successfully demonstrate the internal structure of the lesion and the extent of the tumor. Most tumors appear a diffusely enhancing soft tissue mass on CT. On MRI, these tumors are generally of hypointense to isointense on $T_{1}$ weighted images, of hypointense to hyperintense on $\mathrm{T}_{2}$ weighted images, and exhibit heterogeneous contrast enhancement. Spontaneous tumor haemorrhage and regional metastatic adenopathy have been demonstrated in some cases using MRI or CT, but no evidence of tumoral calcification prior to treatment has been reported. However, the key differences in osseous ES and EES are obvious on CT and MRI. Radiologically, osseous ES is essentially destructive lesion with ill-defined margins and principally involves the medullary cavity. The most common radiologic signs are bone expansion and lytic changes. Although EES is a primary tumor of soft tissue and tends to spread locally, it usually has a pseudocapsule on gross specimen and thus appears wellcircumscribed on CT or MRI. It can cause changes in the cortex of adjacent bone. These radiological characteristics are consistent with those found in our case.
The rarity of EES makes it difficult to ascertain an optimal management for those tumors. However, better results seem to correlate with more aggressive combination of surgery plus radiotherapy plus chemotherapy. Although there is some debate about the management of EES compared with bone ES, it is clear that all members of the ESFT share the propensity for recurrence and metastasis. Treatment is fundamental in the local control of the disease in its primary location, using radical surgery or radiotherapy, and the systemic control of subclinical micrometastasis with chemotherapy. Surgery is the treatment of choice for the local control of the disease if the tumor can be completely resected. The indications for surgery should be carefully considered in each patient with regard to age and location, size, extension, and resectability of the primary tumor, also taking into account the deformity and surgical morbidity that can be produced. EES is radiosensitive. However, the role of radiotherapy is still under debate. In general, radical or adjuvant radiotherapy should be reserved for initially unresectable lesions or residual disease. Rud et al. were the first to report on the importance of multiagent chemotherapy in the treatment of patients with EES [23]. In Whaley's report, combined modality therapy provides excellent local control with reasonable acute and late toxicity [17]. Although the optimum combination chemotherapy is yet to be established, a four drug regimen using vincristine, adriamycin, cyclophosphamide and actinomycin D (VACA) is considered as the standard first-line treatment for patients with localized disease [24]. We used neoadjuvant chemotherapy after confirmation by biopsy to shrink the tumor mass and control possible occult distant metastasis and then radical radiotherapy. He responded well to the regimen of chemotherapy and radiotherapy. Unfortunately, the lesion recurred locally after 10 months, he died of multiple distant metastases 22 months later.

\section{CONCLUSIONS}

EES arising in head and neck is extremely rare. Rapid growth and propensity for recurrence and metastasis are dominant features of this primary malignant soft tissue tumor. It is not easy to diagnose EES by focusing only on histological studies. Although MIC2 is not specific for EES and is expressed in a wide range of unrelated tumors, stain with CD99 provides support for the diagnosis of EES, if used with other features. CT and MRI cannot provide a specific diagnosis, but successfully demonstrate the internal structure of the lesion and the extent of the tumor. The diagnosis of EES in our case was based on clinical, radiological, histological and immunohistochemical findings. Early and confident diagnosis coupled with combined surgical excision of gross 
disease and modern chemotherapy/radiotherapy appears to be the most effective treatment plan.

\section{REFERENCES}

[1] Ewing, J. (1921) Diffuse endothelioma of bone. Proceedings of the New York Pathological Society, 21, 17-24.

[2] Tefft, M., Vawter, G.F. and Mitus, A. (1969) Paravertebral round cell tumors in children. Radiology, 92, 1501-1509.

[3] Angervall, L. and Enzinger, F.M. (1975) Extraskeletal neoplasm resembling Ewing's sarcoma. Cancer, 36, 240-251.

[4] Li, T., Goldberg, R.A., Becker, B. and McCann, J. (2003) Primary orbital extraskeletal Ewing sarcoma. Archives of Ophthalmology, 121, 1049-1052.

doi:10.1001/archopht.121.7.1049

[5] Chao, T.K., Chang, Y.L. and Sheen, T.S. (2000) Extraskeletal Ewing's sarcoma of the scalp. Journal of Laryngology and Otology, 114, 73-75. doi:10.1258/0022215001903744

[6] Lim, T.C., Tan, W.T. and Lee, Y.S. (1994) Congenital extraskeletal Ewing's sarcoma of the face: A case report. Head \& Neck, 16, 75-78. doi:10.1002/hed.2880160115

[7] Iriz, A., Albayrak, L. and Eryilmaz, A. (2007) Extraskeletal primary Ewing's sarcoma of the nasal cavity. International Journal of Pediatric Otorhinolaryngology Extra, 2, 194-197. doi:10.1016/j.pedex.2007.05.008

[8] Özer, C., Arpacı, T., Yıldız, A., Apaydın, F.D., Duce, M.N. and Düzovalı, Ö. (2005) Primary Ewing's sarcoma of the paranasal sinus with intracranial and intraorbital extension. European Journal of Radiology Extra, 55, 47-50.

[9] Boor, A., Jurkovic, I., Friedmann, I., Plank, L. and Kocan, P. (2001) Extraskeletal Ewing's sarcoma of the nose. Journal of Laryngology and Otology, 115, 74-76. doi:10.1258/0022215011906885

[10] Ng, S.H., Ko, S.F., Cheung, Y.C., Wong, H.F. and Jung, S.M. (2004) Extraskeletal Ewing's sarcoma of the parapharyngeal space. British Journal of Radiology, 77, 1046-1049. doi:10.1259/bjr/16676268

[11] Chaudhary, N., Gupta, D., Natesh, V. and Singh, S. (2010) Extraskeletal Ewing's Sarcoma of parapharyngeal space. International Journal of Pediatric Otorhinolaryngology Extra, 5, 159-161. doi:10.1016/j.pedex.2009.08.004

[12] Yang, Y.S. and Hong, K.H. (2004) Extraskeletal Ewing's sarcoma of the larynx. Journal of Laryngology and Otology, 118, 62-64. doi:10.1258/002221504322731682

[13] Kang, M.S., Yoon, H.K., Choi, J.B. and Eum, J.W. (2005) Extraskeletal Ewing's sarcoma of the hard palate. Journal of Korean Medical Science, 20, 687-690. doi:10.3346/jkms.2005.20.4.687

[14] Agir, H., Brasch, H.D. and Tan, S.T. (2007) Extraskeletal Ewing's sarcoma of the submandibular gland. Journal of Plastic, Re-constructive \& Aesthetic Surgery, 60, 13451348. doi:10.1016/j.bjps.2006.01.046

[15] Zachariades, N., Koumoura, F., Liapi-Avgeri, G. and Bouropoulou, V. (1994) Extraskeletal Ewing's sarcoma of the parotid region: A case report with the detection of the tumour's immunophenotypical characteristics. British Journal of Oral and Maxillofacial Surgery, 32, 328-331. doi:10.1016/0266-4356(94)90058-2

[16] Adapa, P., Chung, T.W., Popek, E.J. and Hunter, J.V. (2009) Extraosseous Ewing sarcoma of the thyroid gland. Pediatric Radiology, 39, 1365-1368. doi:10.1007/s00247-009-1388-1

[17] Whaley, J.T., Indelicato, D.J., Morris, C.G., Hinerman, R.W., Amdur, R.J., Mendenhall, W.M., Keole, S.R. and Marcus, R.B. Jr. (2010) Ewing tumors of the head and neck. American Journal of Clinical Oncology, 33, 321326. doi:10.1097/COC.0b013e3181aaca71

[18] Hatori, M., Doi, H., Watanabe, M., et al. (2006) Establishment and characterization of a clonal human extraskeletal Ewing's sarcoma cell line, EES1. Tohoku Journal of Experimental Medicine, 210, 221-230. doi:10.1620/tjem.210.221

[19] Gaona-Luviano, P., Unda-Franco, E., González-Jara, L., Romero, P. and Medina-Franco, H. (2003) Primitive neuroectodermal tumor of the vagina. Gynecologic Oncology, 91, 456-568. doi:10.1016/S0090-8258(03)00500-6

[20] Votta, T.J., Fantuzzo, J.J. and Boyd, B.C. (2005) Peripheral primitive neuroectodermal tumor associated with the anterior mandible: A case report and review of the literature. Oral Surgery, Oral Medicine, Oral Pathology, Oral Radiology and Endodontology, 100, 592-597. doi:10.1016/j.tripleo.2005.03.015

[21] Kowal-Vern, A., Walloch, J., Chou, P., Gonzalez-Crussi, F., Price, J., Potocki, D. and Herman, C.(1992) Flow and image cytometric DNA analysis in Ewing's sarcoma. Modern Pathology, 5, 56-60.

[22] Stuart-Harris, R., Wills, E.J., Philips, J., Langlands, A.O., Fox, R.M. and Tattersall, M.H. (1986) Extraskeletal Ewing's sarcoma: A clinical, morphological and ultrastructural analysis of five cases with a review of the literature. European Journal of Cancer and Clinical Oncology, 22, 393-400. doi:10.1016/0277-5379(86)90104-5

[23] Rud, N.P., Reiman, H.M., Pritchard, D.J., Frassica, F.J. and Smithson, W.A. (1989) Extraosseous Ewing's sarcoma: A study of 42 cases. Cancer, 64, 1548-1553. doi:10.1002/1097-0142(19891001)64:7<1548::AID-CN CR2820640733>3.0.CO;2-W

[24] Antuña García, M.J. (2005) Ewing's sarcoma family of tumors. Clinical and Translational Oncology, 7, 262269. 